\title{
Predictores académicos y sociodemográficos de ansiedad y bienestar psicológico en estudiantes mexicanos de medicina. Estudio transversal
}

\author{
David Luna, ${ }^{1 *}$ Diana Ivette Urquiza-Flores, ${ }^{2}$ Rosa Paola Figuerola-Escoto, ${ }^{3}$ Carolina Carreño-Morales ${ }^{1}$ y \\ Fernando Meneses-González ${ }^{1}$ \\ ${ }^{1}$ Comisión Nacional de Arbitraje Médico, Dirección de investigación; ' 2 nstituto Tecnológico y de Estudios Superiores de Monterrey, Campus Ciudad \\ de México; ${ }^{3}$ Instituto Politécnico Nacional, Centro Interdisciplinario de Ciencias de la Salud Unidad Santo Tomás. Ciudad de México, México
}

\section{Resumen}

Introducción: Estudiantes de medicina reportan mayor ansiedad que estudiantes de otras carreras. El conocimiento sobre su bienestar psicológico es escaso. Objetivo: Identificar factores sociodemográficos y académicos predictores del nivel de ansiedad y bienestar psicológico en estudiantes mexicanos de medicina. Método: Estudio transversal de estudiantes mexicanos de medicina de primer $(n=59)$, tercer $(n=43)$ y quinto semestre $(n=59)$, que contestaron un cuestionario sociodemográfico, la Escala de Ansiedad de Beck, la Escala de Bienestar Psicológico para Adultos y la Escala de Evaluación de la Cohesión y la Adaptabilidad Familiar. Resultados: Las mujeres presentaron mayor ansiedad $(p<0.01)$. La ansiedad en hombres fue similar en los distintos semestres $(p>0.05)$; las mujeres de tercer y quinto semestre fueron más ansiosas que las del primero $(p<0.01)$. Ansiedad y bienestar psicológico correlacionaron negativamente $(p<0.001)$. Se identificaron los subgrupos "Menor ansiedad, mayor bienestar" $y$ "Mayor ansiedad, menor bienestar", y una regresión logística identificó que ser mujer $(O R=4.70)$ y no profesar alguna religión $(O R=2.49)$ son factores predictores de mayor ansiedad. Conclusiones: Las estudiantes de medicina constituyen una población de riesgo para mayor ansiedad y menor bienestar psicológico, lo que compromete su aprendizaje, calidad de vida y futuro ejercicio profesional.

PALABRAS CLAVE: Salud mental. Diferencias entre sexos. Factores predictores. Estudiantes de medicina.

\section{Academic and sociodemographic predictors of anxiety and psychological well-being in Mexican medical students. A cross-sectional study}

\section{Abstract}

Introduction: Medical students report higher levels of anxiety than students from other majors. Knowledge about their psychological well-being is scarce. Objective: To identify sociodemographic and academic factors that predict the level of anxiety and psychological well-being in Mexican medical students. Method: Cross-sectional study of Mexican medical students of first $(n=59)$, third $(n=43)$ and fifth semester $(n=59)$, who answered a sociodemographic questionnaire, Beck Anxiety Inventory, the Psychological Well-being Scale for adults and the Family Adaptability and Cohesion Evaluation Scale. Results: Females showed higher levels of anxiety $(p<0.01)$. Anxiety in males was similar in the different semesters $(p>0.05)$; women of third and fifth semesters were more anxious than those at first semester $(p<0.01)$. Anxiety and psychological well-being were negatively correlated $(p<0.001)$. The "Less anxiety, higher level of well-being" and "More anxiety, lower level of well-being" subgroups were characterized, and a logistic regression identified that being a woman $(O R=4.70)$ and not practicing any religion $(O R=2.49)$ are predictive factors of higher levels of anxiety. Conclusions: Female medical stu- 
dents constitute a population at risk for higher levels of anxiety and less psychological well-being, which compromises their learning, quality of life and future professional practice.

KEY WORDS: Mental health. Gender differences. Predictive factors. Medical students.

\section{Introducción}

Los estudiantes de medicina muestran mayor estrés y prevalencia de psicopatologías más severas en comparación con los estudiantes de otras carreras y de la población general. ${ }^{1-3}$ El estrés puede ocasionar trastornos de ansiedad ${ }^{4}$ y comorbilidad con diversas psicopatologías, incluyendo otros trastornos de ansiedad. ${ }^{5}$ La ansiedad es un sistema de alerta que previene al organismo ante amenazas reales o supuestas. ${ }^{6}$ Su prevalencia en estudiantes de medicina varía entre países, ${ }^{1,7-11}$ aunque algunas condiciones se mantienen constantes: el nivel de ansiedad aumenta en función del desarrollo curricular, ${ }^{2}$ es mayor en mujeres ${ }^{1}$ y entre sus principales fuentes se encuentran aspectos académicos, psicosociales y económicos. ${ }^{12}$

El estrés en estudiantes de medicina impacta negativamente en su bienestar psicológico y potencia el riesgo de psicopatologías. ${ }^{13}$ El bienestar psicológico es un estado afectivo positivo que favorece el funcionamiento óptimo en la vida personal y social, y permite a los individuos percibir control de su vida y entorno..$^{14-16}$ Se considera un aspecto crítico de la formación médica, ${ }^{11}$ aunque se ha reportado su diminución sobre el curso de los estudios en medicina. ${ }^{17}$

Más investigaciones se han enfocado al estudio de la ansiedad en estudiantes de medicina que al bienestar psicológico. ${ }^{18}$ Este estudio identificó factores sociodemográficos y académicos predictores del nivel de ansiedad y bienestar psicológico en estudiantes mexicanos de medicina.

\section{Método}

Estudio transversal. Mediante muestreo no probabilístico por conveniencia se reclutó a estudiantes de medicina inscritos al primer, tercer y quinto semestre. Los criterios de inclusión fueron asistir a una evaluación académica en noviembre de 2018, participar voluntariamente en el estudio y completar cuatro cuestionarios. De 186 estudiantes invitados, 10 no desearon participar.

Se aplicó el cuestionario de variables sociodemográficas y académicas, el Inventario de Ansiedad de Beck (IAB), ${ }^{19}$ la Escala de Bienestar Psicológico para Adultos $(\text { BIEPS-A })^{16}$ y la Escala de Evaluación de la Cohesión y la Adaptabilidad Familiar (FACES-III), ${ }^{20}$ todos validados en población mexicana. ${ }^{21-23}$

El IAB comprende 21 reactivos organizados en cuatro factores: ansiedad subjetiva, ansiedad neurofisiológica, ansiedad autonómica y pánico, que explican $56 \%$ de la varianza $(\alpha=0.93)$. El nivel de ansiedad se consideró de acuerdo con la puntuación: mínimo, 0 a 5 puntos; leve, 6 a 15; moderado, 16 a 30; y severo, 31 a $63 .{ }^{21}$ Se afirmó la presencia de ansiedad con un puntuación $\geq 6$. $^{7}$

La escala BIEPS-A comprende 12 reactivos organizados en cuatro factores: autonomía, proyectos, vínculos y aceptación/control, que explican $60 \%$ de la varianza $(\alpha=0.79$ ). El nivel de bienestar psicológico se consideró promedio cuando la puntuación total fue menor o igual al percentil 50, bajo si fue inferior a este valor o alto si fue mayor o igual al percentil $95 .{ }^{24}$

La escala FACES-III comprende 20 reactivos organizados en dos factores: cohesión y adaptabilidad $(\alpha=0.70)$. Su interpretación de acuerdo con la puntuación para cohesión indicó una familia no relacionada (10 a 34 puntos), semirrelacionada (34 a 40), relacionada (41 a 45) o aglutinada (46 a 50). Para adaptabilidad indicó una familia rígida (10 a 19 puntos), estructurada (20 a 24), flexible (25 a 28) o caótica (29 a 50).

Un investigador solicitó a los estudiantes su colaboración voluntaria en el estudio, explicó sus objetivos y aclaró dudas. A quienes aceptaron participar se les entregó un formato de consentimiento informado y los cuestionarios impresos.

Las diferencias de sexo y semestres respecto a la ansiedad y bienestar psicológico se analizaron mediante ANOVA bifactorial, unifactorial y la prueba post hoc de Tukey. Para evaluar la asociación entre variables se calculó la correlación producto-momento de Pearson y $\chi^{2}$ de independencia. Para estas últimas, con un resultado significativo se calcularon los residuos estandarizados de Pearson como prueba post hoc y la $\mathrm{V}$ de Cramer como indicador del tamaño del efecto. La fuerza de asociación entre variables se interpretó como trivial con valores absolutos $<0.10$, como baja con 0.11 a 0.29 , media con 0.30 a 0.49 y alta $\geq 0.50 .{ }^{25}$

Tras la eliminación de valores atípicos multivariantes se realizó un análisis de conglomerados para crear subgrupos mutuamente excluyentes con los 
Tabla 1. Prevalencia total, por sexo, semestre, del nivel de ansiedad y bienestar psicológico en estudiantes de medicina

\begin{tabular}{|c|c|c|c|c|c|c|c|c|c|}
\hline & $\mathrm{n}$ & $\%$ & $n$ & $\%$ & $n$ & $\%$ & $\chi^{2}$ & $p$ & V \\
\hline \multicolumn{10}{|c|}{ Ansiedad } \\
\hline & \multicolumn{2}{|c|}{ Muestra total } & \multicolumn{2}{|c|}{ Mujeres } & \multicolumn{2}{|c|}{ Hombres } & & & \\
\hline Mínima & 47 & 29.2 & 21 & 18 & 26 & 59.1 & 28.81 & $0.001^{*}$ & 0.43 \\
\hline Leve & 52 & 32.3 & 41 & 35 & 11 & 25 & & & \\
\hline Moderada & 40 & 24.8 & 38 & 32.5 & 2 & 4.5 & & & \\
\hline \multirow[t]{2}{*}{ Severa } & 22 & 13.7 & 17 & 14.5 & 5 & 11.4 & & & \\
\hline & \multicolumn{2}{|c|}{ Semestre 1} & \multicolumn{2}{|c|}{ Semestre 3} & \multicolumn{2}{|c|}{ Semestre 5} & & & \\
\hline Mínima & 25 & 42.4 & 11 & 25.6 & 11 & 18.6 & \multirow[t]{4}{*}{11.26} & \multirow[t]{4}{*}{$0.04^{\star *}$} & \multirow[t]{4}{*}{0.18} \\
\hline Leve & 16 & 27.1 & 14 & 32.6 & 22 & 37.3 & & & \\
\hline Moderada & 12 & 20.3 & 9 & 20.9 & 19 & 32.2 & & & \\
\hline Severa & 6 & 10.2 & 9 & 20.9 & 7 & 11.9 & & & \\
\hline \multicolumn{10}{|c|}{ Bienestar } \\
\hline & \multicolumn{2}{|c|}{ Muestra total } & \multicolumn{2}{|c|}{ Mujeres } & \multicolumn{2}{|c|}{ Hombres } & & & \\
\hline Bajo & 68 & 42.2 & 49 & 41.9 & 19 & 43.2 & 0.98 & $0.61^{\star}$ & 0.07 \\
\hline Medio & 70 & 43.5 & 53 & 45.3 & 17 & 38.6 & & & \\
\hline \multirow[t]{2}{*}{ Alto } & 23 & 14.3 & 15 & 12.8 & 8 & 18.2 & & & \\
\hline & \multicolumn{2}{|c|}{ Semestre 1} & \multicolumn{2}{|c|}{ Semestre 3} & \multicolumn{2}{|c|}{ Semestre 5} & & & \\
\hline Bajo & 26 & 44.1 & 17 & 39.5 & 25 & 42.4 & \multirow[t]{3}{*}{0.30} & \multirow[t]{3}{*}{$0.98^{* \star}$} & \multirow[t]{3}{*}{0.04} \\
\hline Medio & 25 & 42.4 & 20 & 46.5 & 25 & 42.4 & & & \\
\hline Alto & 8 & 13.5 & 6 & 14 & 9 & 15.2 & & & \\
\hline
\end{tabular}

datos de ansiedad y bienestar psicológico. Se empleó una técnica jerárquica con la distancia euclídea al cuadrado y el método de agrupamiento de Ward. La solución se validó mediante un análisis de conglomerados confirmatorio ${ }^{26}$ y los coeficientes gamma, tau-b, tau-c, y d de Somers. ${ }^{27}$ Los subgrupos formados se validaron mediante $t$ de una cola para grupos independientes, con la d de Cohen como índice del tamaño del efecto (efecto pequeño, mediano y grande $d \geq 0.20,0.50$, y 0.80 , respectivamente ${ }^{28}$ ).

Un modelo de regresión logística consideró variable de respuesta la pertenencia al subgrupo "mayor ansiedad, menor bienestar" y como variables predictoras, los datos sociodemográficos y académicos. El modelo se validó por evaluar la hipótesis nula (prueba ómnibus) y su bondad de ajuste (Hosmer-Lemeshow). Se calculó el porcentaje correcto de clasificación de casos, y la $\mathrm{R}^{2}$ de Nagelkerke. Se empleó el programa SPSS, versión 20.

Este estudio fue sometido al Comité de Investigación de la Comisión Nacional de Arbitraje Médico.

\section{Resultados}

Participaron 176 estudiantes de medicina, pero se eliminaron los datos de 15 por haber contestado parcialmente los instrumentos. Los 161 estudiantes retenidos tenían una edad promedio \pm desviación estándar de $19.57 \pm 1.13$ años, $117(72.7 \%)$ mujeres y $44(27.3 \%)$ hombres, 59 cursaban el primer semestre (36.6\%), 43 el tercero $(26.7 \%)$ y 59 el quinto $(36.6 \%)$, 91 en el turno matutino (56.5\%), 38 en el vespertino (23.6) o 32 en horario mixto (19.6\%). El promedio académico al semestre en curso tuvo un rango de 78 a 98 de $100(89.29 \pm 3.91)$ y $12(7.4 \%)$ participantes tuvieron entre una y tres materias reprobadas $(0.09 \pm 0.39)$. La carrera de medicina fue primera opción para 148 (91.9 \%) estudiantes; todos eran solteros, $97(60.2 \%)$ profesaban alguna religión y ocho (5\%) reportaron tener empleo.

El $70.8 \%$ de estudiantes, $82 \%$ mujeres y $40.9 \%$ hombres, presentaron ansiedad en algún nivel de leve a severo. El mayor porcentaje de hombres no 
Tabla 2. Puntuación de ansiedad y bienestar psicológico obtenida por sexo y por semestre en la muestra total, en mujeres y hombres

\begin{tabular}{|c|c|c|c|c|c|c|}
\hline & Media $\pm \mathrm{DE}$ & Media \pm DE & Media \pm DE & & \multirow[t]{2}{*}{$F$} & \multirow[t]{2}{*}{ p } \\
\hline & Muestra total & Mujeres & Hombres & & & \\
\hline Ansiedad & $15.10 \pm 13.49$ & $17.25 \pm 13.07$ & $9.36 \pm 13.04$ & & $15.08^{*}$ & 0.001 \\
\hline \multirow[t]{2}{*}{ Bienestar } & $32.69 \pm 2.65$ & $32.66 \pm 2.65$ & $32.77 \pm 2.70$ & & $0.28^{*}$ & 0.86 \\
\hline & & Semestre 1 & Semestre 3 & Semestre 5 & & \\
\hline \multirow[t]{3}{*}{ Ansiedad } & Muestra total & $11.71 \pm 11.54$ & $17.51 \pm 16.18$ & $16.74 \pm 12.68$ & $1.52^{\star *}$ & 0.22 \\
\hline & Mujeres & $12.26 \pm 10.68$ & $23.81 \pm 16.14$ & $18.47 \pm 11.35$ & $7.75^{\star *}$ & 0.001 \\
\hline & Hombres & $9.76 \pm 14.51$ & $6.87 \pm 9.47$ & $11.66 \pm 15.27$ & $0.51^{* *}$ & 0.59 \\
\hline \multirow[t]{3}{*}{ Bienestar } & Muestra total & $32.74 \pm 2.66$ & $32.90 \pm 2.30$ & $32.49 \pm 2.90$ & $0.40^{\star *}$ & 0.66 \\
\hline & Mujeres & $32.86 \pm 2.49$ & $32.62 \pm 2.40$ & $32.47 \pm 2.97$ & $0.24^{* *}$ & 0.78 \\
\hline & Hombres & $32.30 \pm 3.25$ & $33.37 \pm 2.12$ & $32.53 \pm 2.79$ & $0.63^{* *}$ & 0.53 \\
\hline
\end{tabular}

$\mathrm{DE}=$ desviación estándar. ${ }^{*}$ Comparaciones entre sexos. ${ }^{*}$ Comparaciones entre semestres.

presentó ansiedad (nivel mínimo), mientras que el mayor porcentaje de mujeres la presentó en nivel moderado (Tabla 1). Independientemente del sexo, la mayoría de los estudiantes de primer semestre no presentaron ansiedad (nivel mínimo) (Tabla 1). La puntuación promedio del IAB en hombres fue menor que en mujeres (Tabla 2), quienes también presentaron mayores puntuaciones en el tercer y quinto semestre respecto al primero (Tabla 2). El nivel bajo o promedio de bienestar psicológico fue independiente del sexo o semestre en curso (Tabla 1). No hubo diferencias entre sexos (Tabla 2) o semestres (Tabla 2) en la puntuación promedio en el BIEPS-A. Las puntuaciones totales del IAB y de la escala BIEPS-A mostraron una correlación negativa de intensidad media $(r=-0.32, p<0.001)$.

Antes del análisis de conglomerados, los datos de un participante fueron eliminados debido a que demostraron ser atípicos multivariantes. El análisis de conglomerados jerárquico sugirió una solución de dos subgrupos, validada mediante el análisis confirmatorio, por los coeficientes gamma $=1$, tau-b $=0.95$, tau-c $=0.74$, d de Somers $=0.95$ y por pruebas t. La Tabla 3 muestra las características de los subgrupos "menor ansiedad, mayor bienestar" $(n=116)$ y "mayor ansiedad, menor bienestar" $(n=44)$.

El modelo de regresión logística identificó como factores de riesgo para pertenecer al subgrupo "mayor ansiedad, menor bienestar" ser mujer y no profesar religión. El promedio académico fue un factor protector (Tabla 3). El modelo fue significativo $\left(\chi^{2}=34.95\right.$, $\mathrm{p}=0.02)$, con adecuado ajuste $\left(\chi^{2}=9.35, \mathrm{p}=0.31\right)$, explicó $28.4 \%$ de la varianza y clasificó correctamente $74.4 \%$ de los casos.

\section{Discusión}

El principal resultado de este estudio fue que ser mujer y no profesar religión son predictores de mayor ansiedad y menor bienestar psicológico, siendo el promedio académico un factor protector.

La prevalencia de ansiedad es semejante a la de otros estudios: 57,73 y $79.7 \%{ }^{13,29,30}$ y contrasta con datos nacionales que emplearon el mismo instrumento (IAB), pero que reportaron solo $24.8 \%$ de estudiantes de medicina con ansiedad. ${ }^{8}$ Esto pudo ser debido a diferencias en el criterio diagnóstico utilizado. Consistente con otros trabajos, las mujeres presentaron

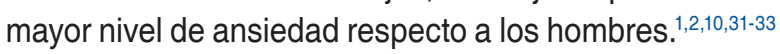
Esta asimetría puede deberse a factores culturales, neurofisiológicos y evolutivos que hace a mujeres más susceptibles de presentar ansiedad. ${ }^{34}$ Un dato novedoso es que la ansiedad en hombres no difirió entre semestres, mientras que las mujeres de semestres avanzados fueron más ansiosas. La más lenta recuperación de la respuesta hormonal al estrés en las mujeres ${ }^{35}$ y la mayor cantidad de estresores académicos en semestres avanzados puede explicar este resultado.

La equivalencia entre sexos en bienestar psicológico es consistente con los datos referidos en la literatura. ${ }^{15}$ En hombres, mantener el mismo nivel de bienestar psicológico y de ansiedad entre semestres indica cierta independencia entre estas variables. ${ }^{36}$ En mujeres, el aumento de ansiedad no disminuyó su bienestar psicológico. Estos datos pueden sugerir un papel protector de la ansiedad que evita la disminución del bienestar psicológico. Al respecto, datos indican que la ansiedad promueve la conducta de aversión al riesgo, ${ }^{37}$ que en el contexto de la educación médica 
Gaceta Médica de México. 2020;156

Tabla 3. Características y predictores sociodemográficos, académicos, y familiares de los estudiantes de acuerdo con su nivel de ansiedad y bienestar psicológico

\begin{tabular}{|c|c|c|c|c|c|c|c|c|c|}
\hline & \multirow{2}{*}{\multicolumn{2}{|c|}{$\begin{array}{c}<\mathrm{A}>\mathrm{B} \\
\text { Media } \pm \mathrm{DE}\end{array}$}} & \multicolumn{2}{|c|}{$>\mathrm{A}<\mathrm{B}$} & \multirow[t]{2}{*}{$\mathbf{p}^{*}$} & \multirow[t]{2}{*}{ d } & \multirow[t]{2}{*}{$\mathbf{R M}$} & \multirow[t]{2}{*}{ IC } & \multirow[t]{2}{*}{$\mathrm{p}^{* * *}$} \\
\hline & & & & $\pm \mathrm{DE}$ & & & & & \\
\hline Total IAB & \multicolumn{2}{|c|}{$7.94 \pm 5.55$} & \multicolumn{2}{|c|}{$33.15 \pm 9.68$} & 0.001 & 3.26 & & & \\
\hline Ansiedad subjetiva & \multicolumn{2}{|c|}{$4.22 \pm 3.31$} & \multicolumn{2}{|c|}{$14.20 \pm 4.74$} & 0.001 & 2.38 & & & \\
\hline Ansiedad neurofisiológica & \multicolumn{2}{|c|}{$2.16 \pm 2.47$} & \multicolumn{2}{|c|}{$10.90 \pm 4.86$} & 0.001 & 2.38 & & & \\
\hline Ansiedad autonómica & \multicolumn{2}{|c|}{$0.66 \pm 1.02$} & \multicolumn{2}{|c|}{$4.29 \pm 2.38$} & 0.001 & 2.14 & & & \\
\hline Pánico & \multicolumn{2}{|c|}{$0.89 \pm 1.16$} & \multicolumn{2}{|c|}{$3.75 \pm 2.07$} & 0.001 & 1.75 & & & \\
\hline Total BIEPS-A & \multicolumn{2}{|c|}{$33.09 \pm 2.31$} & \multicolumn{2}{|c|}{$31.88 \pm 2.85$} & 0.007 & 0.43 & & & \\
\hline Autonomía & \multicolumn{2}{|c|}{$10.24 \pm 1.59$} & \multicolumn{2}{|c|}{$9.68 \pm 1.73$} & 0.05 & 0.30 & & & \\
\hline Proyectos & \multicolumn{2}{|c|}{$11.54 \pm 0.86$} & \multicolumn{2}{|c|}{$11.40 \pm 0.89$} & 0.38 & 0.13 & & & \\
\hline Vínculos & \multicolumn{2}{|c|}{$5.69 \pm 0.56$} & \multicolumn{2}{|c|}{$5.31 \pm 0.95$} & 0.002 & 0.49 & & & \\
\hline Aceptación/control & & $=0.75$ & & 0.84 & 0.33 & 0.15 & & & \\
\hline Edad & & \pm 1.12 & & $=1.15$ & 0.12 & 0.24 & 1.16 & $0.66-2.04$ & 0.59 \\
\hline Promedio académico & & \pm 3.86 & & $=3.61$ & 0.001 & 0.53 & 0.86 & $0.76-0.97$ & 0.01 \\
\hline Materias reprobadas & & $=0.25$ & & 0.60 & 0.01 & 0.40 & 1.36 & $0.40-4.53$ & 0.61 \\
\hline & $n$ & $\%$ & $n$ & $\%$ & $\mathbf{p}^{* *}$ & v & RM & IC & $\mathbf{p}^{* * *}$ \\
\hline & & & & xo & & & & & \\
\hline Mujer & 78 & 67.2 & 38 & 86.4 & 0.01 & 0.19 & 4.70 & $1.55-14.19$ & 0.006 \\
\hline Hombre (R) & 38 & 32.8 & 6 & 13.6 & & & & & \\
\hline & & & & o civil & & & & & \\
\hline Soltero & 116 & 100 & 44 & 100 & NA & NA & & & \\
\hline & & & Pro & religió & & & & & \\
\hline Sí (R) & 76 & 65.5 & 21 & 47.7 & 0.04 & 0.16 & 2.49 & $1.05-5.88$ & 0.03 \\
\hline No & 40 & 34.5 & 23 & 52.3 & & & & & \\
\hline & & & & vaja & & & & & \\
\hline Sí (R) & 5 & 4.3 & 3 & 6.8 & 0.51 & 0.05 & & & \\
\hline No & 111 & 95.7 & 41 & 93.2 & & & 1.20 & $0.19-7.56$ & 0.84 \\
\hline & & & Nivel & lar ma & & & & & \\
\hline Básico o sin información (R) & 5 & 4.3 & 1 & 2.3 & 0.64 & 0.07 & & & \\
\hline Medio superior & 25 & 21.6 & 12 & 27.3 & & & 1.72 & $0.11-25.22$ & 0.69 \\
\hline Superior & 86 & 74.1 & 31 & 70.5 & & & 1.50 & $0.11-19.44$ & 0.75 \\
\hline & & & Nivel & Jar pac & & & & & \\
\hline Básico o sin información (R) & 11 & 9.5 & 1 & 2.3 & 0.22 & 0.13 & & & \\
\hline Medio superior & 10 & 8.6 & 6 & 13.6 & & & 11.32 & $0.71-178.35$ & 0.08 \\
\hline Superior & 95 & 81.9 & 37 & 84.1 & & & 6.62 & $0.57-75.99$ & 0.12 \\
\hline & & & dicin & mo opc & & & & & \\
\hline Sí (R) & 107 & 92.2 & 41 & 93.2 & 0.84 & 0.01 & & & \\
\hline No & 9 & 7.8 & 3 & 6.8 & & & 0.42 & $0.08-2.22$ & 0.30 \\
\hline
\end{tabular}


Tabla 3. Características y predictores sociodemográficos, académicos, y familiares de los estudiantes de acuerdo con su nivel de ansiedad y bienestar psicológico (Continuación)

\begin{tabular}{|c|c|c|c|c|c|c|c|c|c|}
\hline & $n$ & $\%$ & $\mathrm{n}$ & $\%$ & $\mathbf{p}^{* *}$ & v & RM & IC & $\mathbf{p}^{* * *}$ \\
\hline \multicolumn{10}{|c|}{ Semestre } \\
\hline Primero (R) & 47 & 40.5 & 12 & 27.3 & \multirow[t]{3}{*}{0.29} & \multirow[t]{3}{*}{0.12} & & & \\
\hline Tercero & 29 & 25 & 14 & 31.8 & & & 1.17 & $0.33-4.18$ & 0.80 \\
\hline Quinto & 40 & 34.5 & 18 & 40.9 & & & 0.65 & $0.10-4.11$ & 0.65 \\
\hline \multicolumn{10}{|c|}{ Turno } \\
\hline Matutino & 71 & 61.2 & 20 & 45.5 & \multirow[t]{3}{*}{0.17} & \multirow[t]{3}{*}{0.14} & 0.45 & $0.13-1.53$ & 0.20 \\
\hline Vespertino & 25 & 21.6 & 12 & 27.3 & & & 0.80 & $0.18-3.54$ & 0.77 \\
\hline Mixto (R) & 20 & 17.2 & 12 & 27.3 & & & & & \\
\hline \multicolumn{10}{|c|}{ Cohesión } \\
\hline No relacionada $(\mathrm{R})$ & 18 & 15.5 & 7 & 15.9 & \multirow[t]{4}{*}{0.28} & \multirow[t]{4}{*}{0.15} & & & \\
\hline Semirelacionada & 21 & 18.1 & 14 & 31.8 & & & 2.15 & $0.53-8.60$ & 0.27 \\
\hline Relacionada & 39 & 33.6 & 12 & 27.3 & & & 1.13 & $0.28-4.44$ & 0.85 \\
\hline Aglutinada & 38 & 32.8 & 11 & 25 & & & 0.91 & $0.22-3.75$ & 0.90 \\
\hline \multicolumn{10}{|c|}{ Adaptabilidad } \\
\hline Rígida (R) & 6 & 5.2 & 2 & 4.5 & \multirow[t]{4}{*}{0.97} & \multirow[t]{4}{*}{0.03} & & & \\
\hline Estructurada & 16 & 13.8 & 7 & 15.9 & & & 0.72 & $0.06-8.75$ & 0.79 \\
\hline Flexible & 24 & 20.7 & 8 & 18.2 & & & 0.34 & $0.02-4.38$ & 0.40 \\
\hline Caótica & 70 & 60.3 & 27 & 61.4 & & & 0.54 & $0.04-6.17$ & 0.62 \\
\hline
\end{tabular}

podría prevenir la toma de decisiones impulsivas en el cuidado de la salud las cuales podrían comprometer el bienestar psicológico en mujeres médicos. Es posible que con mayores niveles de ansiedad, las mujeres estudiantes de medicina planeen de mejor manera las conductas y decisiones a tomar respecto del cuidado de la salud de sus pacientes, lo cual ayude a mantener estable su bienestar psicológico.

Consistente con lo anterior, la fuerza de asociación entre ansiedad y bienestar psicológico reportada indica cierto grado de independencia entre dichas variables. Esto sugiere que intervenir en una de ellas no necesariamente afectará en forma intensa la otra.

Una aportación importante del estudio fue el describir el perfil de estudiantes de medicina con "menor ansiedad, mayor bienestar" y "mayor ansiedad, menor bienestar". El primero agrupó mayormente hombres que profesan alguna religión, con mayor promedio académico y menor número de materias reprobadas; el segundo incluyó mayormente mujeres que no profesan religión, con menor promedio y mayor número de materias reprobadas. No obstante, la regresión logística no logró retener el número de materias reprobadas como factor predictor de pertenencia al segundo perfil. Profesar religión puede tener una función protectora a la ansiedad, que eleva la calidad de vida y el rendimiento académico y, por consiguiente, el promedio académico. Una asociación semejante fue argumentada previamente. ${ }^{38}$

La diferencia entre perfiles radica principalmente en el nivel de ansiedad, cuya discrepancia en la puntuación total y por factor del IAB mostró un tamaño de efecto grande. Respecto a la puntuación en la escala BIESP-A, la equivalencia en los factores "proyectos y aceptación/control" indica que, en general, los estudiantes evaluados tienen definidas sus metas y le dan sentido a su vida, a la vez que se aceptan como son y perciben control de sus circunstancias. ${ }^{16}$ No obstante, el segundo perfil mostró menor bienestar general, menor capacidad para tomar decisiones de forma independiente (autonomía), y para establecer relaciones sociales empáticas (vínculos). Esto último es importante pues dichos estudiantes en un futuro deberán establecer relaciones empáticas con sus pacientes, lo 
cual se ha demostrado favorece la calidad de la atención a la salud. ${ }^{39}$ Además, existen datos que demuestran el efecto protector del apoyo social sobre el riesgo de desarrollar trastornos de ansiedad en mujeres. ${ }^{40}$

Una limitación de este estudio fue recolectar los datos durante una evaluación semestral. Esto pudo ocasionar un sesgo en los niveles de ansiedad. No obstante, los datos obtenidos son consistentes con la literatura, lo cual supone la menor influencia de dicho evento. Se recomienda realizar estudios transversales y longitudinales para evaluar ansiedad y bienestar psicológico en diferentes momentos del semestre a fin de verificar su estabilidad o tendencia.

\section{Conclusiones}

Las mujeres estudiantes de medicina presentan mayor ansiedad y menor bienestar psicológico, lo que compromete su calidad de vida, aprendizaje y futuro ejercicio profesional. Se requieren estrategias de apoyo y resiliencia para estudiantes de medicina en general y para mujeres en particular. ${ }^{41}$

\section{Financiamiento}

De la Comisión Nacional de Arbitraje Médico, además este estudio forma parte del proyecto de investigación con registro 20195546, por el Instituto Politécnico Nacional.

\section{Bibliografía}

1. Darby LN, Thomas MR, Shanafelt TD. Systematic review of depression, anxiety, and other indicators of psychological distress among U.S. and Canadian medical students. Acad Med. 2006;81:354-373.

2. Segura ANA, Eraña RIE, Luna-de-la-Garza MV, Castorena-Ibarr J, López CMV. Análisis de la ansiedad en los primeros encuentros clínicos: experiencias utilizando la simulación clínica en estudiantes de pregrado. Educ Med. 2019.

3. Perales A, Sogi C, Morales R. Estudio comparativo de salud mental en estudiantes de medicina de dos universidades estatales peruanas. An Fac Med. 2003;64:239-246.

4. Ko SM, Kua EH, Fones CS. Stress and the undergraduates. Singapore Med J. 1999:40:627-630.

5. Wehrenberg M, Prinz SM. The anxious brain. EEUU: Norton; 2007.

6. DSM-5. Manual diagnóstico y estadístico de los trastornos mentales. EEUU: American Psychiatric Publishing: 2013.

7. De Oliveira TF, Fuganti-De Souzal M, Pfützenreuterl G, Carriero LV, Traebertl E, Traebertl J. Prevalence of anxiety and depression among medical students of private university. Rev Bras Educ Med. 2015;39:388-394.

8. Santillán TTC, Hernández PMR, Bravo Y, Castro HA, Romero M. Prevalencia de síntomas de ansiedad en una muestra de estudiantes universitarios en una facultad de ciencias de la salud en México. Tesis Psicol. 2016:11:12-22.

9. Sarkar S, Gupta R, Menon V. A systematic review of depression, anxiety, and stress among medical students in India. J Mental Health Hum Behav. 2017:22:88-96

10. Ogunsemi OO, Afe T, Odusan O, Oguntona SA, Adefuye BO, Oyelekan AAA. Psychological well-being of medical students in a state university, Nigeria. Res J Health Sci. 2013;1:90-98.
11. Dunn LB, Iglewicz A, Moutier C. A conceptual model of medical student well-being: promoting resilience and preventing burnout. Acad Psychiatry. 2008;32:44-53.

12. Castillo-Pimienta C, Chacón-De la Cruz T, Díaz-Véliz G. Ansiedad y fuentes de estrés académico en estudiantes de carreras de la salud. Inv Ed Med. 2016;5:230-237.

13. Mosley TH, Perrin SG, Neral SM, Dubbert PM, Grothues CA, Pinto BM, Stress, coping and well-being among third-year medical students. Acad Med. 1994;69:765-767.

14. Deci EL, Ryan RM. Hedonia, eudaimonia, and well-being: an introduction. J Happiness Stud. 2008;9:1-11.

15. Udhayakumar $\mathrm{P}$, Illango $\mathrm{P}$. Psychological Wellbeing among college students. J Soc Work Educ. 2018;3:79-89.

16. Casullo M. Evaluación del bienestar psicológico en Iberoamérica. Argentina: Paidós; 2002.

17. Park CL, Adler NE. Coping style as a predictor of health and well-being across the first year of medical school. Health Psychol. 2003;22:627-631.

18. Aboalshamat K, Hou XY, Strodl E. Psychological well-being status among medical and dental students in Makkah. Saudi Arabia: a cross-sectional study. Med Teach. 2015;37:S75-S81.

19. Beck AT, Epstein N, Brown G, Steer RA. An Inventory for measuring clinical anxiety: psychometric properties. J Consult Clin Psychol. 1988:56:893-897.

20. Olson DH. Circumplex model of marital and family systems: Assessing family functioning. En: Walsh $\mathrm{F}$ (editor). Normal family processes. EEUU: Guilford Press; 1993.

21. Robles R, Varela R, Jurado S, Páez F. Versión mexicana del inventario de ansiedad de Beck: propiedades psicométricas. Rev Mex Psicol. 2011;8:211-217.

22. Luna D. Figuerola-Escoto RP, López-Cruz H. Propiedades psicométricas de la Escala de Bienestar Psicológico para Adultos (BIEPS-A) en universitarios mexicanos. Trabajo de investigación presentado en: 3er Congreso Internacional de Psicología. La psicología ante los desafíos de construir vertientes para el mejoramiento de la sociedad; 2019, Ciudad de México, México.

23. Ponce-Rosas ER, Gómez-Clavelina FJ, Terán-Trillo M, Irigoyen-Coria AE Landgrave-Ibáñez S. Validez de constructo del cuestionario FACES III en español (México). Aten Primaria. 2002;30:624-630.

24. Páramo MA, Straniero CM, García CS, Torrecilla NM, Gómez EE. Bienestar psicológico, estilos de personalidad y objetivos de vida en estudiantes universitarios. Pen Psicol. 2012;10:7-21.

25. Ellis PD. The essential guide to effect sizes: An introduction to statistical power, meta-analysis and the interpretation of research results. Reino Unido: Cambridge University Press; 2010.

26. Fisher L, Ransom DC. An empirically derived typology of families: I. Relationships with adult health. Fam Process. 1995;34:161-182.

27. Göktas A, Isçi Ö. A comparison of the most commonly used measures of association for doubly ordered square contingency tables via simulation. Metodoloski Zvezki. 2011;8:17-37.

28. Aron A, Aron E. Estadística para psicólogos. Argentina: Prentice Hall; 2002.

29. Inam SN, Saqib A, Alam E. Prevalence of anxiety and depression among medical students of private university. J Pak Med Assoc. 2003;53:44-47.

30. Aboalshamat K, Jawhari A, Alotibi S, Alzahrani K, Al-Mohimeed H, Alzahrani $\mathrm{M}$, et al. Relationship of self-esteem with depression, anxiety, and stress among dental and medical students in Jeddah, Saudi Arabia. JIMD. 2017;4:61-68.

31. Sánchez-Marín C, Chichón-Peralta J, Leon-Jimenez F, Alipazaga-Pérez P. Trastornos mentales en estudiantes de medicina humana en tres universidades de Lambayeque, Perú. Rev Neuropsiquiatr. 2016;79:197-206.

32. Reyes-Carmona C, Monterrosas-Rojas AM, Navarrete-Martínez A, Acosta-Martínez EP, Torruco-García U. Ansiedad de los estudiantes de una facultad de medicina mexicana, antes de iniciar el internado. Inv Ed Med. 2017:6:42-46.

33. Guerrero-López JB, Heinze-Martin G, Ortiz-De León S, Cortés-Morelos J, Barragán-Pérez V, Flores-Ramos M. Factores que predicen depresión en estudiantes de medicina. Gac Med Mex. 2013:149:598-604.

34. Hines M. Brain gender. EEUU: Oxford; 2005.

35. Olff M, Langeland W, Draijer N, Gersons BP. Gender differences in posttraumatic stress disorder. Psychol Bull. 2007;133:183-204.

36. Sandoval BS, Dorner PA, Véliz BA. Bienestar psicológico en estudiantes de carreras de la salud. Inv Ed Med. 2017;24:260-266.

37. Kirsch M, Windmann S. The role of anxiety in decision-making. Rev Psychol. 2009;16:19-28.

38. Ashouri FP, Rasekhi SA. Review on Medical Students Mental Health Problems and Proposed Solutions. IEJM. 2015;4:23-31.

39. Kim SS, Kaplowitz S, Johnston MV. The effects of physician empathy on patient satisfaction and compliance. Eval Health Prof. 2004;27:237-251.

40. Kendler KS, Myers J, Prescott CA. Sex differences in the relationship between social support and risk for major depression: a longitudinal study of opposite-sex twin pairs. Am J Psychiatry. 2005;162:250-256.

41. Hamza MA, Abdulaziz AA, Ebrahim S, Gominda GP, Eiad AA. Stress and its effects on medical students: a cross-sectional study at a college of medicine in Saudi Arabia. J Health Popul Nutr. 2011;29:516-522. 